\title{
Monitoring quantum jumps
}

\section{A neat theoretical calculation shows how to monitor the state of a simple model atom without interfering with its quantum state. But Heisenberg's principle remains intact.}

IF Heisenberg's uncertainty principle means anything at all, it must surely mean that it is impossible to keep a continuous record of the transition of a quantum system, say an atom, between two distinct quantum states. That is the general expectation. Suppose, for the sake of argument, that a particular atom may be found in one of only two states, a ground state and an excited state, and that some measurement is carried out to establish which state is occupied. The result is certain to be that the measurement will interfere with the system. Ordinarily the atom will be left in the condition that the measurement reveals, at least until something happens to shift it into the other state. So an ambition to monitor the state of the atom continuously would seem to conflict with these familiar expectations.

But, it seems the outlook may not be that unpromising. Richarch J. Cook of the United States Air Force Institute of Technology at Dayton, Ohio, and H.J. Kimble of the University of Texas at Austin, have devised what seems to be a neat way around the difficulty (Phys. Rev. Lett. 54, 1023; 1985). Their proposal hangs on the techniques by means of which it has now become possible to confine single atoms (strictly, ions) in a small region of space by means of electromagnetic traps of various kinds (of which the Penning trap is the prototype). Spectroscopists nurse ambitions to use such traps as ways of carrying out spectroscopy with single atoms. Cook and Kimble's proposal for the direct observation of quantum jumps is one such application. To be fair, the authors do cautiously use the word "possibility" in the title of their paper. They go on to add that the effect which they predict has not yet been observed, "but probably will be in the near future".

This is how the proposed Cook/Kimble experiments would work. The first need is for an atom with two excited states which are connected to the ground state by transitions that are respectively strong and weak. Put one such atom into a Penninglike trap (which is naturally easier said than done) and illuminate it with a laser with a frequency which corresponds to the excitation energy between the ground state and the strongly coupled excited state, and measure the intensity of the fluorescence radiation of the same frequency which is given off by the single atom. What happens is merely that the atom is repeatedly excited (by the laser beam) and de-excited (by spontaneous radiation emission). If the ground and excited states are indeed strongly coupled, the rate of emission of fluorescence radiation can be as much as 100 million photons a second, well within the capacity of radiation detectors. And while, as Cook and Kimble say, the fluorescent signal will in reality be a succession of quantum events, there will be no difficulty in recording this as steady current in, say, a photomultiplier system. That is the first step.

Next, take a laser whose frequency corresponds to the energy difference between the ground state and the other excited state, that to which the ground state is only weakly coupled, and simultaneously illuminate the single atom with that as well. The predominant process will be the repeated excitation and de-excitation of the strongly coupled state, but occasionally the weak excitation will be excited instead. And when that happens, the electron whose oscillation into and out of the strongly coupled state is responsible for the main fluorescence signal will be out of action, marooned in the weakly coupled state instead. And since the coupling is weak, the main fluorescence signal will be turned off for the length of time the electron stays in the weakly coupled state. So the output from the photomultiplier circuit will consist of a series of signals corresponding to the full value of the strong fluorescence punctuated by gaps when the signal vanishes, and which correspond to the times when the weakly excited state is occupied. That, then, is the running record of when the weakly excited state is occupied. The signal from the photomultipliers will be, for all practical purposes, like telegraph signals in which the dots and dashes have random length. There will be no difficuly in interpreting the record with macroscopic measuring devices - the output signal can be drawn on a moving paper chart and interpreted by eye, if need be.

Cook and Kimble attribute the origin of their neat proposal to $\mathrm{H}$. Dehmelt, who is said to have suggested a scheme rather like this in the context of the detection and measurement of weakly coupled optical transitions. Their own chief purpose is to provide a theoretical calculation of the details of the effects of competitive excitation within their three-level model atom on the fluorescent signal eventually produced, and in particular to calculate the statistical properties of the gaps in the fluorescence signal in terms of the parameters describing the two transitions.

The argument follows a now familiar line. The rate of excitation of each state is determined by the Einstein parameters from each transition, that which determines the rate of spontaneous emission (conventionally labelled $A$ ) and that which determines (with the intensity of the exciting radiation) the rate of excitation (labelled $B)$. Cook and Kimble simplify the algebra somewhat by supposing that the light exciting the strongly coupled transition is intense enough to saturate the single atom (so that, if the weakly coupled excitation did not exist, the atom would spend exactly half of its time in the ground state and the excited states). The practical problem is merely to calculate the probability that the fluorescence signal due to the strong transition will persist for a length of time, say $T$. Inevitably, the results all depend on the Einstein parameters for each transition, but their practical value is that they involve the Einstein $A$ parameter representing the rate of spontaneous de-excitation of the weakly-coupled state, a quantity that is not easily determined by conventional measurements.

This neat recipe for an experiment not yet carried out will obviously spur on the single-atom spectroscopists. On the assumption that the result is that which has now been predicted, the question obviously arises of the sense in which Heisenberg's uncertainty principle must be qualified. Fortunately, a little reflection will show that the question is a red herring. The underlying assumption in what Cook and Kimble have done is that the rate of transition between the ground state and the strongly coupled excited state is very much greater than the rate of transition to the weakly-coupled state. Moreover, there is no way in which this system could be used for arranging that the atom is excited one way or the other to order, for the state of the atom at any time is determined by strictly random processes.

So despite this apparent assault, the uncertainty principle remains intact. What does, however, emerge from this neat piece of experimental design is a vivid illustration of the interesting things that will be possible when single-atom spectroscopy moves into the laboratory, which should not be much delayed.

John Maddox 